\title{
A NOTE ON INTEGRAL CLOSURE
}

\author{
JUDITH SALLY
}

ABSTRACT. Let $R$ be an integrally closed domain and $x_{i}, y_{j}$ $(1 \leqq i \leqq n, 1 \leqq j \leqq m) R$-sequences. Let

$$
T=R\left[x_{1}^{\alpha_{1}} \cdots x_{n}^{\alpha_{n}} / y_{1}^{\beta_{1}} \cdots y_{m}^{\beta_{m}}\right],
$$

where the $\alpha_{i}$ and $\beta_{j}$ are positive integers. If $T$ is integrally closed then

$$
\alpha_{1}=\cdots=\alpha_{n}=1 \text { or } \beta_{1}=\cdots=\beta_{m}=1 \text {. }
$$

(*) is sufficient for $T$ to be integrally closed in the following cases:

(1) $R$ is Noetherian and the $\left(x_{i}, y_{j}\right) R$ are distinct prime ideals,

(2) $R$ is a polynomial ring over an integrally closed domain and the $x_{i}$ and $y_{j}$ are indeterminates.

It is known ([2], [3]) that the monoidal transform of a domain $R$ with respect to an ideal $I$ is normal (i.e., integrally closed) if high powers of $I$ are complete, and that the converse holds provided that $R$ is Noetherian. However, in most instances, the criterion of completeness is not very practical for proving integral closure. This paper is concerned with the simplest case, namely the preservation of integral closure of a domain $R$ upon adjunction of a quotient $a / b$ of certain elements of $R$. The condition on $a, b$ is symmetric so this work may alternately be viewed as an investigation of the completeness of the ideals $(a, b)^{k}$ for all large $k$. I would like to thank the referee for a number of helpful suggestions.

The following notation will be fixed throughout. Let $R$ be an integrally closed domain. Let $x_{i}, y_{j}(1 \leqq i \leqq n, 1 \leqq j \leqq m)$ be $R$-sequences and let

$$
T=R\left[x_{1}^{\alpha_{1}} x_{2}^{\alpha_{2}} \cdots x_{n}^{\alpha_{n}} / y_{1}^{\beta_{1}} y_{2}^{\beta_{2}} \cdots y_{m}^{\beta_{m}}\right],
$$

where the $\alpha_{i}$ and $\beta_{j}$ are positive integers.

Proposition 1. If $T$ is integrally closed, then

$$
\alpha_{1}=\alpha_{2}=\cdots=\alpha_{n}=1 \text { or } \beta_{1}=\beta_{2}=\cdots=\beta_{m}=1 \text {. }
$$

Proof. Assume $\alpha_{i}>1$ and $\beta_{j}>1$ for some $i$ and $j$. Then

$$
\left(x_{1}^{\alpha_{1}} \cdots x_{i}^{\alpha_{i}-1} \cdots x_{n}^{\alpha_{n}} / y_{j}\right)^{2}=\left(x_{1}^{\alpha_{1}} \cdots x_{i}^{\alpha_{i}-2} \cdots x_{n}^{\alpha_{n}}\right)\left(x_{1}^{\alpha_{1}} \cdots x_{i}^{\alpha_{i}} \cdots x_{n}^{\alpha_{n}} / y_{j}^{2}\right)
$$

Received by the editors September 17, 1971 and, in revised form, January 26, 1972. AMS 1970 subject classifications. Primary 13B20, 13E05, 14A05.

Key words and phrases. Integrally closed domain, Noetherian domain, $R$-sequence, monoidal transform, complete ideal.

(c) American Mathematical Society 1972 
is an element of $T$. To contradict the fact that $T$ is integrally closed we must show that $x_{1}^{\alpha_{1}} \cdots x_{i}^{\alpha_{i}-1} \cdots x_{n}^{\alpha_{n}} / y_{j}$ is not in $T$. Suppose it is. Then we have

$$
\begin{aligned}
x_{1}^{\alpha_{1}} \cdots x_{i}^{\alpha_{i}-1} \cdots x_{n}^{\alpha_{n}} / y_{j}= & r_{0}+r_{1}\left(x_{1}^{\alpha_{1}} \cdots x_{n}^{\alpha_{n}} / y_{1}^{\beta_{1}} \cdots y_{m}^{\beta_{m}}\right) \\
& +\cdots+r_{k}\left(x_{1}^{\alpha_{1}} \cdots x_{n}^{\alpha_{n}} / y_{1}^{\beta_{1}} \cdots y_{m}^{\beta_{m}}\right)^{k},
\end{aligned}
$$

where the $r_{q}$ are in $R$ and $k \geqq 1$. Thus,

$$
\begin{aligned}
y_{1}^{k \beta_{1}} \cdots y_{j}^{k \beta_{j}-1} \cdots & y_{m}^{k \beta_{m}}\left(x_{1}^{\alpha_{1}} \cdots x_{i}^{\alpha_{i}-1} \cdots x_{n}^{\alpha_{n}}\right)=r_{0}\left(y_{1}^{\beta_{1}} \cdots y_{m}^{\beta_{m}}\right)^{k} \\
& +r_{1}\left(x_{1}^{\alpha_{1}} \cdots x_{n}^{\alpha_{n}}\right)\left(y_{1}^{\beta_{1}} \cdots y_{m}^{\beta_{m}}\right)^{k-1}+\cdots+r_{k}\left(x_{1}^{\alpha_{1}} \cdots x_{n}^{\alpha_{n}}\right)^{k} .
\end{aligned}
$$

It follows that $r_{0}=r_{0}^{\prime}\left(x_{1}^{\alpha_{1}} \cdots x_{i}^{\alpha_{i}-1} \cdots x_{n}^{\alpha_{n}}\right)$, for some $r_{0}^{\prime} \in R$. Hence,

$$
\begin{aligned}
\left(1-r_{0}^{\prime} y_{j}\right)\left(y_{1}^{k \beta_{1}} \cdots\right. & \left.y_{j}^{k \beta_{j}-1} \cdots y_{m}^{k \beta_{m}}\right) \\
& =x_{i}\left(r_{1} y_{1}^{\beta_{1}} \cdots y_{m}^{\beta_{m}}+\cdots+r_{k}\left(x_{1}^{\alpha_{1}} \cdots x_{n}^{\alpha_{n}}\right)^{k-1}\right) .
\end{aligned}
$$

This gives the contradiction $1 \in\left(x_{i}, y_{j}\right) R$.

Note that the adjunction of a quotient $x_{1} / y_{1}$ of irreducible $R$-sequence elements is not sufficient for the integral closure of $R\left[x_{1} / y_{1}\right]$. (For example, let $K$ be a field, $x, y$ indeterminates and $R=K\left[x, y, x^{2} / y\right]_{P}$, where $P=$ $\left(x, y, x^{2} / y\right)$. If $x_{1}=x^{2} / y$ and $y_{1}=y$, then $R\left[x_{1} / y_{1}\right]$ is not integrally closed.) In the Noetherian case, with an additional hypothesis on the ideals $\left(x_{i}, y_{j}\right) R$, we have a converse to Proposition 1.

THEGREM 2. Assume in addition that $R$ is Noetherian and that the ideals $\left(x_{i}, y_{j}\right) R$ are distinct prime ideals. Then $(*)$ is sufficient for $T$ to be integrally closed.

Remarks. (i) If $R$ is a polynomial ring over a field and the $x_{i}$ and $y_{j}$ are indeterminates, then the statement of the theorem is easily checked using the Jacobian criterion.

(ii) Under the further assumption that $x_{i}, y_{1}, \cdots, y_{m}$ and $y_{j}, x_{1}, \cdots$, $x_{n}(1 \leqq i \leqq n, 1 \leqq j \leqq m)$ are $R$-sequences, $\mathrm{R}$. Fossum has given a direct proof of the fact that the ideals

$$
\left(x_{1}^{\alpha_{1}} \cdots x_{n}^{\alpha_{n}}, y_{1} \cdots y_{m}\right)^{k}, \quad\left(x_{1} \cdots x_{n}, y_{1}^{\beta_{1}} \cdots y_{m}^{\beta_{n}}\right)^{k}
$$

are complete for all $k$. It then follows from ([2], [3]) that $T$ is integrally closed.

Proof of TheOREM 2. Since any domain is the intersection of its localizations at maximal primes of principal ideals [1, Theorem 53], we will show that all such localizations of $T$ are integrally closed. Let $Q$ be a prime ideal of $T$. If $y_{j} \notin Q$ for all $1 \leqq j \leqq m$, then, since $T \subset R\left[1 / y_{1}^{\beta_{1}} \cdots y_{m}^{\beta_{m}}\right]$, we have that $T_{Q}=R_{Q \cap R}$ and $T_{Q}$ is, therefore, integrally closed. Suppose 
that $y_{j} \in Q$ for some $j$. We use the fact that

$$
T=R[t] /\left(y_{1}^{\beta_{1}} \cdots y_{m}^{\beta_{m}} t-x_{1}^{\alpha_{1}} \cdots x_{n}^{\alpha_{n}}\right),
$$

where $t$ is an indeterminate [1, p. 102, Exercise 3]. Let $Q^{\prime}$ denote the inverse image of $Q$ in $R[t]$. Now $y_{j} \in Q$ implies that $\left(x_{i}, y_{j}\right) R \subseteq Q \cap R$ for some $i$. We distinguish two cases. First, assume that $Q^{\prime}=\left(x_{i}, y_{j}\right) R[t]$ so that $Q=\left(x_{i}, y_{j}\right) T$. Note that none of the elements $x_{1}, \cdots, x_{i-1}$, $x_{i+1}, \cdots, x_{n}, y_{1}, \cdots, y_{j-1}, y_{j+1}, \cdots, y_{m}, x_{1}^{\alpha_{1}} \cdots x_{n}^{\alpha_{n}} / y_{1}^{\beta_{2}} \cdots y_{m}^{\beta_{m}}$ are in $Q$. If $\alpha_{1}=\cdots=\alpha_{n}=1$, then $Q T_{Q}=y_{j} T_{Q}$. If some $\alpha_{k}>1$ but $\beta_{1}=\cdots=\beta_{m}=1$, then $Q T_{Q}=x_{i} T_{Q}$. Thus $(*)$ implies that $T_{Q}$ is a discrete valuation ring (DVR). (Note that if $T$ is Macaulay, e.g. if $R$ is Macaulay, the proof is finished since we have shown that $T_{Q}$ is a DVR for all rank 1 primes.) To complete the proof, we consider the case $Q^{\prime} \supsetneqq\left(x_{i}, y_{j}\right) R[t]$. In this case $Q^{\prime} /\left(x_{i}, y_{j}\right) R[t]$ is a prime ideal of rank $\geqq 1$ in the domain

$$
R[t] /\left(x_{i}, y_{j}\right) R[t] \text {. }
$$

It follows that $Q^{\prime}$ contains an $R$-sequence of length 3. Thus $Q$ contains an $R$-sequence of length 2 and cannot belong to a principal ideal.

If $R$ is not Noetherian, one might drop down to a Noetherian subring $R_{0}$. However, in general, the ideals $\left(x_{i}, y_{j}\right) R_{0}$ will not be prime. This technique will work in the following case.

COROllary 3. Let $R=S\left[x_{1}, \cdots, x_{n}, y_{1}, \cdots, y_{m}\right]$, where $S$ is an integrally closed domain and the $x_{i}$ and $y_{j}$ are indeterminates. Then $(*)$ is sufficient for $T$ to be integrally closed.

Proof. Let $z=f / g$ with $f, g \in R$. Suppose that there is an equation $z^{k}+t_{1} z^{k-1}+\cdots+t_{k}=0$, where $t_{i} \in T, 1 \leqq i \leqq k$. Each $t_{i}$ is a polynomial in $x_{1}^{\alpha_{1}} \cdots x_{n}^{\alpha_{n}} / y_{1}^{\beta_{1}} \cdots y_{m}^{\beta_{m}}$ with coefficients $h_{i 0}, \cdot: \cdot h_{i d_{i}}$ in $R$. Let $S_{0}$ be the prime integral domain of $S$. Let $S_{1}$ be the ring generated over $S_{0}$ by the coefficients of $f$ and $g$ and the coefficients of all the $h_{i j}, 1 \leqq i \leqq k, 1 \leqq j \leqq d_{i}$. $S_{1} \subseteq S$. Let $R_{1}=S_{1}^{\prime}\left[x_{1}, \cdots, x_{n}, y_{1}, \cdots, y_{m}\right]$, where $S_{1}^{\prime}$ is the integral closure of $S_{1}$ (in its quotient field). $S_{1}^{\prime} \subseteq S$ so that the $x_{i}$ and $y_{j}$ are indeterminates over $S_{1}^{\prime} . R_{1}$ is Noetherian since $S_{1}^{\prime}$ is $[4,(37.5),(35.3)]$. Now $z$ is integral over $T_{1}=R_{1}\left[x_{1}^{\alpha_{1}} \cdots x_{n}^{\alpha_{n}} / y_{1}^{\beta_{1}} \cdot y_{m}^{\beta_{m}}\right]$. By Theorem 2, $z \in T_{1} \subseteq T$. Thus, $T$ is integrally closed.

We conclude with a remark concerning the general case of a monoidal transform of a domain $R$ with respect to an arbitrary ideal $I$. In [5] it is proved that $I^{k}$ is complete for all $k$ if the following conditions are satisfied: (1) $R$ is integrally closed, (2) $\bigcap_{j=0}^{\infty} I^{j}=0,(3) G_{I}(R)$, the associated graded ring of $R$ with respect to $I$, is a domain. Actually, the proof uses (1), (2) and the fact that $G_{I}(R)$ contains no nilpotent elements so that (3) may be replaced by $\left(3^{\prime}\right) G_{I}(R)$ is reduced. Thus, for example, if $R$ is an integrally 
closed domain, and $I$ is a radical ideal which is generated by an $R$-sequence $a_{1}, \cdots, a_{m}$, and satisfies $\bigcap_{j=0}^{\infty} I^{j}=0$, then $I^{k}$ is complete for all $k$. By ([2], [3]) the monoidal transform of $R$ with respect to $I$ is normal.

\section{BIBLIOGRAPHY}

1. I. Kaplansky, Commutative rings, Allyn and Bacon, Boston, Mass., 1970. MR 40 \#7234.

2. J. Lipman, Rational singularities, with applications to algebraic surfaces and unique factorization, Inst. Hautes Études Sci. Publ. Math. No. 36 (1969), 195-279. MR 43 \#1986.

3. A. Mattuck, Complete ideals and monoidal transforms, Proc. Amer. Math. Soc. 26 (1970), 555-560. MR 42 \#272.

4. M. Nagata, Local rings, Interscience Tracts in Pure and Appl. Math., no. 13, Interscience, New York, 1962. MR 27 \#5790.

5. G. Scheja, Über ganz-algebraische Abhängigkeit in der Idealtheorie, Comment. Math. Helv. 45 (1970), 384-390. MR 42 \#7468.

Department of Mathematics, University of Chicago, Chicago, Illinois 60637

Current address: Department of Mathematics, Northwestern University, Evanston, Illinois 60201 